\title{
Depletion of the neural precursor cell pool by glucocorticoids
}

Shuang Yu, MD, PhD ${ }^{1^{*}}$, Alexandre V. Patchev, MD ${ }^{1}$, Yan Wu, MD, PhD ${ }^{1 \dagger}$, Jie Lu, MD, PhD ${ }^{1 \ddagger}$, Florian Holsboer, $\mathrm{MD}, \mathrm{PhD}^{1}$, Jing-Zhong Zhang, $\mathrm{MD}, \mathrm{PhD}^{2}$, Nuno Sousa, $\mathrm{MD}, \mathrm{PhD}^{3}$

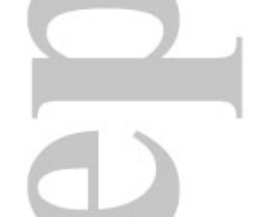
and Osborne F.X. Almeida, PhD ${ }^{1}$

${ }^{1}$ Max Planck Institute of Psychiatry, Kraepelinstrasse 2-10, 80804 Munich, Germany ${ }^{2}$ Beijing Institute for Neuroscience, Capital Medical University, Beijing 100069, P.R. China

${ }^{3}$ Life and Health Sciences Research Institute (ICVS), University of Minho, 4710-057 Braga, Portugal

${ }^{\dagger}$ Present address: Department of Anatomy, Capital Medical University, Beijing 100054, P.R. China

‡ Present address: Department of Neurology, Harvard Medical School, Boston, MA 02215, USA

Correspondence to: Dr. Shuang Yu and Dr. Osborne F.X. Almeida Max Planck Institute of Psychiatry, Kraepelinstrasse 2, 80804 Munich, Germany Tel: (+49) 89-30622-216; Fax: (+49) 89-30622-461;

E-mail:shuang@mpipsykl.mpg.de; osa@mpipsykl.mpg.de

Running head: Glucocorticoid depletion of neural precursors

Keywords: apoptosis; glucocorticoid; hippocampus; neural precursors; neurogenesis

Number of characters in the title / running head (with spaces): 62/45

Number of words in the abstract / the manuscript body: 242/2874

Number of color figures: 6 


\section{Abstract}

Objective: Glucocorticoids (GC) are indicated for a number of conditions in obstetrics and perinatal medicine; however, the neurodevelopmental and long-term neurological consequences of early-life GC exposure are still largely unknown. Preclinical studies have demonstrated that GC have a major influence on hippocampal cell turnover by inhibiting neurogenesis and stimulating apoptosis of mature neurons. Here we examined the fate of the limited pool of neural progenitor cells (NPC) after GC administration during neonatal development; the impact of this treatment on hippocampal structure was also studied.

Methods: Phenotype-specific genetic and antigenic markers were used to identify cultured NPC at various developmental stages; the survival of these cells was monitored after exposure to the synthetic glucocorticoid dexamethasone (DEX). In addition, the effects of neonatal DEX treatment on the neurogenic potential of the rat hippocampus were examined by monitoring the incorporation of bromodeoxyuridine and expression of Ki67 antigen at various postnatal ages.

Results: Multipotent nestin-expressing NPC and Ta1-tubulin-expressing immature neurons succumb to GC-induced apoptosis in primary hippocampal cultures. Neonatal GC treatment results in marked apoptosis among the proliferating population of cells in the dentate gyrus, depletes the NPC pool, and leads to significant and sustained reductions in the volume of the dentate gyrus.

Interpretation: Both NPC and immature neurons in the hippocampus are sensitive to the pro-apoptotic actions of GC. Depletion of the limited NPC pool during early life retards hippocampal growth, thus allowing predictions about the potential neurological and psychiatric consequences of neonatal GC exposure. 


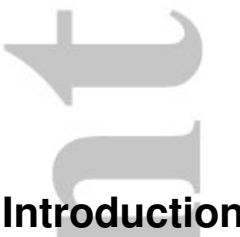

Acquisition and loss of hippocampal neurons are implicated in the regulation of cognition, mood and neuroendocrine function. ${ }^{1-4}$ Most likely, the availability of hippocampal neurons determines neuroplastic changes in the intra-hippocampal circuitry as well as connectivity between the hippocampus and other cortical and sub-cortical areas. ${ }^{5}$ The subgranular zone (SGZ) of the hippocampal dentate gyrus is endowed with a pool of neural precursor cells (NPC) which can divide and differentiate into either neurons or glial cells. ${ }^{2,6}$ Newly-generated neurons integrate into existing hippocampal circuits ${ }^{6}$ and facilitate learning and memory. ${ }^{2-4}$ Neurogenesis tapers off over lifetime and is regulated by intrinsic (e.g. age ${ }^{7,9}$ ) and extrinsic signals (e.g. stress ${ }^{10,11}$ ) whose actions are mainly mediated by GC. Since the size of the NPC pool is a potentially important determinant of life-long hippocampal function, there is considerable interest in the link between lifetime neurogenesis and cognitive deficits that result from exposure to high GC levels. ${ }^{12,13}$

The synthetic glucocorticoid receptor (GR) agonist, dexamethasone (DEX), is commonly used in obstetrics and neonatal medicine. Previously, we demonstrated that DEX induces cell cycle arrest ${ }^{14}$ and apoptosis $^{15-17}$ in mature neurons of the dentate gyrus. In this study, we addressed the question of whether DEX can directly influence the survival of NPC. In addition, we tested the hypothesis that neonatal DEX administration permanently depletes the neurogenic pool. Our results show that GC target NPC for apoptosis and that neonatal GC markedly reduces the number of NPC available for the generation of new neurons.

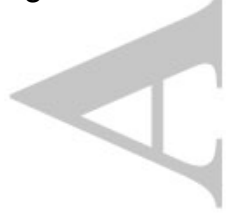




\section{Materials and methods}

Drugs and plasmids: Dexamethasone (DEX; Fortecortin ${ }^{\mathrm{TM}}$; Merck, Darmstadt, Germany), was added to cultures for $48 \mathrm{~h}$ (24 h after transfection). The GR antagonist, RU38486 (10 $\mu \mathrm{M}$; NHPP, Torrance, CA) was added $1 \mathrm{~h}$ before DEX application. Mitotic cells were labeled $(24 \mathrm{~h})$ with 5-bromo-2'-deoxyuridine (BrdU; $20 \mu \mathrm{M}$; Sigma, St. Louis, MO). The specific caspase 3 and 9 inhibitors, Ac-DEVD-cmk $(1 \mu \mathrm{M})$ and Ac-LEHD-cmk (30 $\mu \mathrm{M})$, were obtained from Calbiochem (Schwalbach, Germany) and applied 30 min before addition of DEX.

Neural precursor cells (NPC), neural progenitors and astrocytes were labeled with pBSIISK-E/Nestin-EGFP, ${ }^{18}$ pBSII SK-Ta1-GFP ${ }^{19}$, and pGFAP-GFP ${ }^{20}$ (courtesy of Drs. Hideyuki Okano, Freda Miller, and Helmut Kettenmann, respectively) .

Primary hippocampal cultures and transfection: Hippocampal cultures were prepared from Wistar rats (Charles River, Sulzfeld, Germany) on postnatal day (PND) 4, and transfected ( 10\% efficiency) 5 days after plating. ${ }^{16}$

Animals and tissues: EU and NIH guidelines on animal care and experimentation were observed. Forty-eight male Wistar rats were housed under standard laboratory conditions. Rats received s.c. injections of either vehicle (saline) or DEX on PND1-7 (DEX $200 \mu \mathrm{g} / \mathrm{kg} / \mathrm{d}$ on PND1-3; $100 \mu \mathrm{g} / \mathrm{kg} / \mathrm{d}$ on PND 4-7). All animals received a single i.p. injection of $\mathrm{BrdU}(50 \mathrm{mg} / \mathrm{kg}) 24 \mathrm{~h}$ before killing on PND10, 18 or 28 . Serial coronal cryosections $(20 \mu \mathrm{m})$, extending over the entire length of the hippocampal formation, were cut and mounted before sequential double-staining of every $8^{\text {th }}$ section with antibodies against BrdU (1:200; DAKO, Hamburg, Germany) and Ki67 (1:500, Biotrend, Cologne, Germany); cell nuclei were counterstained with Hoechst $33342(1 \mu \mathrm{g} / \mathrm{ml}, 10 \mathrm{~min})$. 
Immunocyto- and histochemistry: Cells or sections were fixed (4\% paraformaldehyde), permeabilized (0.3\% Triton-X100/PBS), blocked and incubated $\left(4^{\circ} \mathrm{C}\right)$ with anti- BrdU (after treatment with 2 N HCl), nestin (1:1000; Millipore, Goettingen, Germany), -TuJ1 (1:500; Babco, Richmond, CA), -MAP2 (1:500; Sigma), -doublecortin (1:500; Santa Cruz, Heidelberg, Germany), -GFAP (1:1500; DAKO or 1:4,000, Sigma), -NeuN (1:500; Millipore), -O4 (1:500; Millipore), -GR (1:300; M20, Santa Cruz), -Sox2 (1:300, Santa Cruz), cleaved caspase 3 (1:200; Cell Signaling/NEB, Frankfurt, Germany) and p47-phox (1:200, Millipore). Immunoreactivity was visualized using appropriate Alexa Fluor-conjugated secondary antibodies (1:500; Invitrogen). Cells were analyzed (ImagePro software, Media Cybernetics, Bethesda, MD) on an Olympus BX-60 microscope. Cell counts were performed on 10 individual microscopic fields $\left(0.072 \mathrm{~mm}^{2}\right)$, randomly chosen across two diameters of each coverslip (400X magnification). An average of 1,000 cells or 100 transfected cells was sampled on each coverslip; results shown represent values from 6-9 coverslips/treatment.

Apoptotic cells were identified by TUNEL histochemistry (with FITC- or Texas Red-conjugated avidin; Vector, Burlinghame, CA), immunostaining for cleaved (active) caspase 3, or Hoechst 33342 staining. Those cells showing morphologocal signs of DNA fragmentation ${ }^{17,21}$ were considered to be apoptotic.

Stereology: Stereolnvestigator (MicroBrightField, Williston/VT) was used to estimate the volumes of different subdivisions of the dentate gyrus and cell densities $\left(N_{V}\right)$ in the subgranular zone (SGZ) of the dentate gyrus. The total number of $\mathrm{BrdU}^{+}$or $\mathrm{Ki} 67^{+} \mathrm{NPC}$ in the SGZ was derived from the product of Nv and total SGZ volume. To identify BrdU and Ki67 double-stained cells, sections were examined (XY, YZ and XZ views) by confocal microscopy (Olympus IX81 LSM, Hamburg, Germany). 
Statistics: Numerical data (shown as mean \pm SEM) were subjected to 2-tailed Student's $t$-tests or ANOVA and appropriate post-hoc analysis (SPSS Inc, Chicago, IL). The level of significance was preset at $\mathrm{p}<0.05$.

\section{Results}

\section{Phenotypic identity and GR expression in hippocampal cultures}

After 6 days in vitro (DIV), hippocampal cultures expressed markers specific to NPC ( 40\% nestin $\left.{ }^{+}\right)$and immature neurons $\left(\sim 35 \% \mathrm{TuJ}^{+}\right.$and $\left.\mathrm{DCX}^{+}\right)$; approximately $10 \%$ of the cells were young neurons $\left(\mathrm{NeuN}^{+}\right)$, and $15 \%$ were astrocytes (glial fibrillary acidic protein [GFAP] $]^{+}$) or oligodendrocytes $\left(\mathrm{O}^{+}\right)(\mathrm{Fig}$ 1A). Immunoreactive GR was detectable in NPC and immature/young neurons (Fig 1B and Fig. 1C). GR expression was observed in $\sim 45 \%$ of nestin-GFP-transfected NPC and $\sim 65 \%$ of Ta-tubulin-GFP labeled immature neurons (Fig 1B); stimulation with DEX resulted in translocation of immunoreactive GR to the nucleus, suggestive of its transcriptional potential (Fig 1B, inset). Treatment of cultures with DEX resulted in a dose-dependent induction of apoptosis that was preventable by pre-treatment with the GR antagonist RU38486 (Fig 1D); since consistently robust effects were observed at a dose of $10^{-5}$ $\mathrm{M}$, this dose was chosen for all subsequent in vitro experiments.

\section{Regulation of NPC and post-mitotic hippocampal cell fate by GC}

Neuroplasticity depends on the availability of NPC. ${ }^{6}$ While neurogenesis is implicated in recovery from stroke, ${ }^{22}$ reduced proliferative capacity of hippocampal cells is associated with epilepsy, ${ }^{23}$ impaired 
cognition $^{2}$ and depression. ${ }^{24,25}$ We show here that DEX reduces the number of immunocytochemically identified NPC (by $\sim 39 \%)$, neuroblasts $(\sim 39 \%)$ and immature neurons $(\sim 54 \%)(P<0.05$, in all cases;

Fig. 2A).

We previously demonstrated that GC induce apoptosis in hippocampal cells in culture ${ }^{16,17}$ and that GC-induced apoptosis in situ is prominent in the SGZ where NPC reside and proliferate. ${ }^{21,26}$ To examine the hypothesis that apoptosis leads to a reduction in NPC and immature neuron numbers, we next treated hippocampal cultures with the cytosine analog bromodeoxyuridine ( $\mathrm{BrdU}$, to reveal recently proliferated cells $^{27}$ ) and DEX for $24 \mathrm{~h}$ before quantifying apoptosis (identified by TUNEL or activated caspase 3 immunoreactvity) in recently-proliferated (BrdU-labeled ${ }^{27}$ ) cells. As compared to untreated cells (Fig 2B1-2B5), DEX-treated cells showed greater co-localization of BrdU and TUNEL signals (Fig 2C1-2C5). Evaluation of individual merged images revealed significantly increased apoptosis among both mitotic (BrdU': CON: $8.4 \pm 3.4 \%$; DEX: $17.8 \pm 5.0 \%$; $\mathrm{P}<0.05)$ and resting (BrdU: CON: $27.6 \pm$ 3.0\%; DEX: $41.0 \pm 4.1 \% ; \mathrm{P}<0.05)$ cell populations after GC treatment (Fig 2D). The results obtained with TUNEL histochemistry were corroborated by cleaved (activated) caspase 3 immunocytochemistry (Fig 2E).

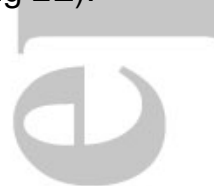

\section{Phenotype-specificity of the apoptotic actions of GC}

NPC proliferate and differentiate along either neuronal or glial lineages. ${ }^{6}$ Given the intrinsic characteristics of NPC and the heterogeneous nature of primary hippocampal cultures (Fig 1), we here analyzed the cell phenotypes targeted for GC-induced apoptosis in mixed hippocampal cultures transfected with specific plasmids that would facilitate distinction between NPC (nestin-EGFP) and 
neuronal progenitors (Ta1-GFP). Exposure of cells to DEX produced a significant increase in TUNEL-labeled apoptotic cells among the NPC (Fig 3A-C, 3G-I and 3U; P < 0.05) and neuronal progenitor (Fig 3D-F, 3J-L and 3U; P < 0.05) cell populations; the TUNEL results were confirmed by staining for cleaved (activated) caspase 3 immunoreactivity (Fig 3M-P, 3Q-T and 3V; $\mathrm{P}<0.05$ ). In all cases, the apoptotic actions of DEX were attenuated when cells were pre-treated with the GR antagonist RU 38486, indicating their mediation by GR (Fig 3U, 3V). Interestingly, astrocytes marked with GFAP-GFP did not succumb to the apoptotic effects of DEX (Fig 3U).

\section{Mitochondrial mechanisms mediate GC-induced apoptosis in NPC}

The data showing that DEX treatments leads to an activation of caspase 3 (Fig 2E, Fig 3M-3T, 3U, $3 \mathrm{~V}$, and Fig 5B-D) in NPC hinted at involvement of the mitochondrial or 'instrinsic' pathway of apoptosis. ${ }^{15}$ These findings were confirmed in hippocampal cultures using pharmacological inhibitors of caspase 3 and its upstream caspase, caspase 9 (Fig 4A). Examining events upstream of the caspases, we observed that DEX treatment dose-dependently increases the ratio of pro-apototic bax to anti-apoptotic $b c l-2$ mRNA expression, without influencing the bax:bcl $\left.\right|_{x L}$ mRNA expression ratio (Fig 4B); the latter findings are consistent with the fact that the predominant anti-apoptotic protein in developing neurons is $\mathrm{BCl}-2$ rather than $\mathrm{Bcl}_{\mathrm{XL}}{ }^{26}$

The mitochondrial proteins $\mathrm{Bax}$ and $\mathrm{Bcl}-2$ act in a rheostatic manner to regulate the integrity of the mitochondrial permeability transition which is particularly sensitive to perturbation by reactive oxygen species (ROS). Measurement of ethidium intercalation into DNA showed that ROS generation represents a mechanism through which DEX induces apoptosis (Fig 4C). Additionally, DEX stimulates 
ROS production in NPC (Fig 4D and Fig 4E); this effect is accompanied by a translocation of membrane-associated $\mathrm{p} 47^{\text {phox }}$ (an essential component of the nicotinamide adenine dinucleotide phosphate $[\mathrm{NADPH}]$ oxidase [Nox] complex required for the production of superoxide anions) (Fig 4F) and reductions in the activities of $\mathrm{Cu}^{++} / \mathrm{Zn}^{++}$superoxide dismutase and glutathione, two key anti-oxidant enzymes (Fig 4G).

\section{Depletion of the NPC pool by GC treatment during peak neurogenesis in vivo}

Hippocampal neurogenesis occurs at a high frequency during early postnatal life. ${ }^{28}$ However, NPC have limited self-renewal capacity ${ }^{29}$ and the NPC pool from which new neurons are generated diminishes exponentially with age; ${ }^{7} 9 \mathrm{GC}$ are thought to at least partially contribute to the latter phenomenon. ${ }^{8}$ On the other hand, proliferating hippocampal cells were previously reported to express GR only sparsely. ${ }^{30}$ As shown in Fig $5 A$, numerous cells in the neonatal dentate gyrus express GR along a gradient that increases from the SGZ to the inner layers of the GCL where more mature granule neurons are localized. Importantly, DEX treatment provoked a 60\% increase in apoptosis (increase in active caspase 3 immunoreactivity) in the SGZ (Fig 5B-D; P < 0.05). Two types of NPC are found in the SGZ: quiescent neural precursors (QNP; GFAP-positive, proliferate relatively slowly) and amplifying neural precursors (ANP; GFAP-negative, display high proliferative activity). ${ }^{31}$ Accordingly, it was considered important to investigate if QNP and ANP might be differentially sensitive to glucocorticoids. Both QNP and ANP express nestin and Sox2, but whereas nestin levels in ANP diminish over time, Sox2 expression is maintained at relatively steady levels in both NPC subtypes and serves as a more reliable marker of NPC (QNP: GFAP+/Sox2+; ANP: GFAP-/Sox2+). Exploiting these characteristics, 
co-localization studies showed that a similar proportion of QNP and ANP express GR (Suppl Fig S1A-S1C), suggesting their similar vulnerability to DEX.

We subsequently assessed the impact of neonatal GC administration on the proliferative capacity of the dentate gyrus in later life by performing stereological counts of the number of BrdU- and Ki67-stained cells in the SGZ of PND10, 18 and 28 days (Fig 6A-C). The results of this analysis revealed that neonatal treatment with DEX results in a significant reduction in the number of cells available for mitosis at any given time $(P<0.05$; Fig $6 D)$, suggesting a depletion of the NPC pool by neonatal DEX. Interestingly, the absolute differences between the number of proliferating cells in the SGZ of both control and DEX-treated rats diminished over time (but remained significantly different), probably reflecting age-related decreases in proliferative activity (Fig $6 \mathrm{D}$ ) and the fact that a sub-population of NPC that do not express GR (see Suppl Fig S1) may escape the apoptotic actions of neonatal GC treatment.

Since expansion of the GCL occurs primarily during early postnatal life, we next carried out a stereological assessment of the volumes of the SGZ and GCL. This analysis revealed that SGZ volumes of DEX-treated animals were significantly smaller $(P<0.05)$, despite similar volumetric increments over time (14-20\%) (Fig 6E); the latter suggests proliferation by residual NPC that were spared from the apoptotic effects of neonatal DEX. While both controls and DEX-treated animals showed significant increases in the volumes of their GCL between the ages of 10 and 28 days (Fig 6E, $P<0.05)$, GCL volumes in the DEX-treated animals were significantly smaller than in controls (Fig 6E, $P<0.05)$. In sum, these results suggest that reduced neurogenesis and subsequently, reduced cell acquisition in the GCL, result in a marked retardation of GCL development in animals exposed to neonatal DEX (Fig 6E). 


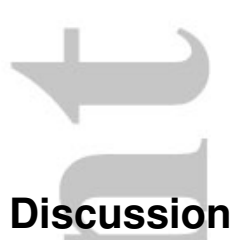

Several neurological and psychiatric disorders are hallmarked by hippocampal dysfunction. The last decade has witnessed compelling evidence for a link between hippocampal function and cell turnover in the postnatal hippocampus. ${ }^{6,13,24,25,32}$ Neuronal turnover in the hippocampus is a dynamic process involving neurogenesis and apoptosis in the germinative layer (SGZ) of the dentate gyrus; ${ }^{3}$ stress and elevated GC levels inhibit neurogenesis and stimulate apoptosis in the hippocampus. ${ }^{11,12,21,}$ ${ }^{26}$ While GC are known to interfere with the neural cell cycle, ${ }^{14}$ it is not known whether GC target NPC for apoptosis. Accordingly, we here examined the incidence of apoptosis in hippocampal cultures that were genetically marked with developmental phase-specific markers to identify proliferating multipotent NPC and NPC destined to become neurons. In addition, we studied the consequences of neonatal treatment with DEX, a synthetic GC (when neurogenesis and apoptosis occur at high frequency ${ }^{28}$ ) on dentate gyrus development in situ.

The presented results demonstrate that both NPC and neuronal progenitors are subject to DEX-induced apoptosis. The actions of DEX were shown to be mediated by GR which are expressed by NPC, neuronal progenitors and mature neurons (in culture) and by QNP and ANP cells residing in the SGZ; notably, the SGZ displays a prominent apoptotic response to DEX. It is important to note, however, that since GR expression by NPC is not ubiquitous, a sub-population of NPC may be (at least transiently) spared from the actions of DEX. However, given the finite self-renewing properties of $\mathrm{NPC},{ }^{29}$ disruption of the lifelong cycle of neuronal birth and therefore, sustained deleterious effects on hippocampal growth and function, is a plausible scenario being investigated in a long-term study. Meanwhile, analysis of the mechanisms through which DEX induces NPC apoptosis revealed a role for the mitochondrial pathway. Consistent with previous findings, ${ }^{33}$ our results indicate that the pro-apoptotic 
actions of DEX are initiated by an increase in ROS levels and concomitant decreases in the cellular defenses against oxidative stress. Through their disruption of the mitochondrial membrane potential, these events subsequently lead to activation of caspase 9 and caspase 3. Notably, we show that DEX treatment results in increased activation of the 'executor caspase', caspase 3, in NPC in culture and in the SGZ of the intact hippocampus.

The capacity of the hippocampus to produce new neurons declines markedly with age. ${ }^{7-9,34}$ While previous work reported an $80 \%$ decrease in the neurogeneic capacity of the hippocampus between 1 and 22 months of age, ${ }^{7-9,34}$ our results show an even steeper decline ( 92\%) between PND10 and 28. Thus, the hippocampus undergoes its most dynamic structural organization during the early postnatal period, with a precipitous depletion of the NPC pool ${ }^{9}$ that probably reflects changes in the milieu that normally encourages NPC proliferation. ${ }^{35}$ Given that NPC are vulnerable to the apoptotic actions of DEX (this study), and have a limited capacity for self-renewal, ${ }^{29}$ as well as the fact that the dentate gyrus increases in neuronal number and volume for at least 1 year, ${ }^{36}$ it was considered important to examine whether DEX influences the in vivo NPC pool in a transient or sustained fashion. We observed that neonatal DEX treatment induces a sustained reduction in the number of mitotic cells and, importantly, retards the volumetric growth of the SGZ and GCL. This finding is consistent with previous reports in rats and rhesus monkeys. ${ }^{37-39}$ On the other hand, postnatal neurogenesis and granule cell volumes appear to be unaltered by prenatal exposure to $\mathrm{DEX}^{40}$ and neurogenesis is only transiently inhibited when DEX is administered to adults. ${ }^{41}$ These observations suggest that early postnatal life may represent a window during which NPC are particularly sensitive to DEX and that exposure to DEX during this period results in a protracted retardation of dentate gyrus development. 
The paradigm of chronic DEX administration during perinatal life is clinically relevant; there is convincing evidence that glucocorticoids during early childhood lead to impairments of neuromotor functions and cognition, as well as head and somatic growth.$^{42,}{ }^{43}$ This study shows that the hippocampus endures increased levels of neuronal apoptosis, retarded growth and sustained reductions in the rate of neurogenesis when DEX is administered during neonatal life; moreover, an earlier study associated such treatment with reduced forebrain expression of synaptic proteins and disruption of the ontogeny of neurotransmitter systems. ${ }^{37}$ Since lifetime cognitive performance relies on plasticity (including neurogenesis) in the hippocampus, ${ }^{2,4,6,34}$ the sustained depletion of the NPC pool by neonatal DEX is likely to have a major impact on lifetime learning and memory. Lastly, early-life experiences that stimulate endogenous glucocorticoid secretion and interfere with neuroplasticity are established etiopathogenic factors in a number of psychiatric conditions, best exemplified by major depression. ${ }^{1,13}$

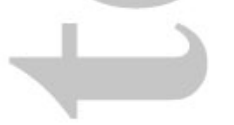

\section{Acknowledgements}

We thank Dieter Fischer, Jutta Waldherr and Rainer Stoffel for technical assistance, Dr. Peter Hutzler for help with confocal microscopy, and Carola Hetzel for administrative help. S. Yu, Y. Wu and J. Lu were supported by fellowships from the Max Planck Society. This study was partly supported by grants from the German Academic Exchange Service (DAAD) and the Portuguese Rectors' Conference (CRUP), a grant from Gulbenkian Foundation (JG 0495), and an Integrated Project grant from the European Commission (CRESCENDO, Contract No. LSHM-CT-2005-01852). 


\section{References}

1. Sousa N, Cerqueira JJ, Almeida OFX. Corticosteroid receptors and neuroplasticity. Brain Res Rev 2008;57:561-570.

2. Shors TJ. From stem cells to grandmother cells: how neurogenesis relates to learning and memory. Cell Stem Cell 2008;3:253-258.

3. Dupret D, Fabre A, Döbrössy MD, et al. Spatial learning depends on both the addition and removal of new hippocampal neurons. PLoS Biol 2007;5:e214.

4. Dalla C, Bangasser DA, Edgecomb C, et al. Neurogenesis and learning: acquisition and asymptotic performance predict how many new cells survive in the hippocampus. Neurobiol Learn Mem 2007;88:143-148.

5. Bessa JM, Ferreira D, Melo I, et al. The mood-improving actions of antidepressants do not depend on neurogenesis but are associated with neuronal remodeling. Mol Psychiatry (in press).

6. Zhao C, Deng W, Gage FH. Mechanisms and functional implications of adult neurogenesis. Cell 2008;132:645-660.

7. Kuhn HG, Dickinson-Anson H, Gage FH. Neurogenesis in the dentate gyrus of the adult rat: age-related decrease of neuronal progenitor proliferation. J Neurosci 1996;16:2027-2033.

8. Cameron HA, McKay RD. Restoring production of hippocampal neurons in old age. Nat Neurosci 1999;2:894-897.

9. Olariu A, Cleaver KM, Cameron HA. Decreased neurogenesis in aged rats results from loss of granule cell precursors without lengthening of the cell cycle. J Comp Neurol 2007;501:659-667.

10. Gould E, Tanapat P, McEwen BS, et al. Proliferation of granule cell precursors in the dentate gyrus of adult monkeys is diminished by stress. Proc Natl Acad Sci USA 1998;95:3168-3171. 
11. Wong EY, Herbert J. Raised circulating corticosterone inhibits neuronal differentiation of progenitor cells in the adult hippocampus. Neuroscience 2006;137:83-92.

12. Cerqueira JJ, Mailliet F, Almeida OFX, et al. The prefrontal cortex as a key target of the maladaptive response to stress. J Neurosci 2007;27:2781-2787.

13. Lupien SJ, McEwen BS, Gunnar MR, et al. Effects of stress throughout the lifespan on the brain, behaviour and cognition. Nat Rev Neurosci 2009;10:434-445.

14. Crochemore C, Michaelidis TM, Fischer D, et al. Enhancement of p53 activity and inhibition of neural cell proliferation by glucocorticoid receptor activation. FASEB J 2002;16:761-770.

15. Yu S, Holsboer F, Almeida OFX. Neuronal actions of glucocorticoids: focus on depression. J Steroid Biochem Mol Biol 2007;108:300-309.

16. Lu J, Goula D, Sousa N, et al. Ionotropic and metabotropic glutamate receptor mediation of glucocorticoid-induced apoptosis in hippocampal cells and the neuroprotective role of synaptic N-methyl-D-aspartate receptors. Neuroscience 2003;121:123-131.

17. Crochemore $\mathrm{C}, \mathrm{Lu} \mathrm{J}, \mathrm{Wu} \mathrm{Y}$, et al. Direct targeting of hippocampal neurons for apoptosis by glucocorticoids is reversible by mineralocorticoid receptor activation, Mol Psychiatry 2005;10:790-798.

18. Kawaguchi A, Miyata T, Sawamoto K, et al. Nestin-EGFP transgenic mice: visualization of the self-renewal and multipotency of CNS stem cells. Mol Cell Neurosci 2001;17:259-273.

19. Wang $\mathrm{S}$, Wu H, Jiang J, et al. Isolation of neuronal precursors by sorting embryonic forebrain transfected with GFP regulated by the T alpha 1 tubulin promoter. Nat Biotechnol 1998;16:196-201.

20. Nolte C, Matyash M, Pivneva T, et al. GFAP promoter-controlled EGFP-expressing transgenic mice: a tool to visualize astrocytes and astrogliosis in living brain tissue. Glia 2001;33:72-86. 
21. Hassan AH, von Rosenstiel P, Patchev VK, et al. Exacerbation of apoptosis in the dentate gyrus of the aged rat by dexamethasone and the protective role of corticosterone. Exp Neurol 1996;140:43-52.

22. Carmichael ST. Themes and strategies for studying the biology of stroke recovery in the poststroke epoch. Stroke 2008;39:1380-1388.

23. Parent JM. Adult neurogenesis in the intact and epileptic dentate gyrus. Prog Brain Res 2007;163:529-540.

24. Kempermann G, Krebs J, Fabel K. The contribution of failing adult hippocampal neurogenesis to psychiatric disorders. Curr Opin Psychiatry 2008;21:290-295.

25. Banasr M, Duman RS. Keeping 'trk' of antidepressant actions. Neuron 2008;59:349-351.

26. Almeida OFX, Condé GL, Crochemore C, et al. Subtle shifts in the ratio between pro- and antiapoptotic molecules after activation of corticosteroid receptors decide neuronal fate. FASEB J $2000 ; 14: 779-790$

27. Bauer S, Patterson PH. The cell cycle-apoptosis connection revisited in the adult brain. J Cell Biol $2005 ; 171: 641-650$.

28. Altman J, Das GD. Autoradiographic and histological evidence of postnatal hippocampal neurogenesis in rats. J Comp Neurol 1965;124:319-335.

29. Crane JF, Trainor PA. Neural crest stem and progenitor cells. Annu Rev Cell Dev Biol 2006;22:267-86.

30. Garcia A, Steiner B, Kronenberg G, et al. Age-dependent expression of glucocorticoid- and mineralocorticoid receptors on neural precursor cell populations in the adult murine hippocampus. Aging Cell 2004;3:363-371. 
31. Segi-Nishida E, Warner-Schmidt JL, Duman RS. Electroconvulsive seizure and VEGF increase the proliferation of neural stem-like cells in rat hippocampus. Proc Natl Acad Sci USA 2008;105:11352-11357.

32. Chrousos GP, Kino T. Glucocorticoid action networks and complex psychiatric and/or somatic disorders. Stress 2007;10:213-219.

33. McIntosh LJ, Hong KE, Sapolsky RM. Glucocorticoids may alter antioxidant enzyme capacity in the brain: baseline studies. Brain Res 1998;791:209-214.

34. Heine VM, Maslam S, Joëls M, et al. Prominent decline of newborn cell proliferation, differentiation, and apoptosis in the aging dentate gyrus, in absence of an age-related hypothalamus-pituitary-adrenal axis activation. Neurobiol Aging 2004;25:361-375.

35. Hattiangady B, Shetty AK. Aging does not alter the number or phenotype of putative stem/progenitor cells in the neurogenic region of the hippocampus. Neurobiol Aging 2008;29:129-147.

36. Bayer SA, Yackel JW, Puri PS. Neurons in the rat dentate gyrus granular layer substantially increase during juvenile and adult life. Science 1982;216:890-892.

37. Kreider ML, Tate CA, Cousins MM, et al. Lasting effects of developmental dexamethasone treatment on neural cell number and size, synaptic activity, and cell signaling: critical periods of vulnerability, dose-effect relationships, regional targets, and sex selectivity. Neuropsychopharmacology 2006;31:12-35.

38. Uno H, Eisele S, Sakai A, et al. Neurotoxicity of glucocorticoids in the primate brain. Horm Behav 1994;28:336-348. 
39. Coe CL, Kramer M, Czéh B, et al. Prenatal stress diminishes neurogenesis in the dentate gyrus of juvenile rhesus monkeys. Biol Psychiatry 2003;54:1025-1034.

40. Tauber SC, Bunkowski S, Schlumbohm C, et al. No long-term effect two years after intrauterine exposure to dexamethasone on dentate gyrus volume, neuronal proliferation and differentiation in common marmoset monkeys. Brain Pathol 2008;18:497-503.

41. Kim JB, Ju JY, Kim JH, et al. Dexamethasone inhibits proliferation of adult hippocampal neurogenesis in vivo and in vitro. Brain Res 2004;1027:1-10.

42. Yeh TF, Lin YJ, Lin HC, et al. Outcomes at school age after postnatal dexamethasone therapy for lung disease of prematurity. N Engl J Med 2004;350:1304-1313.

43. Baud O, Sola A. Corticosteroids in perinatal medicine: how to improve outcomes without affecting the developing brain? Semin Fetal Neonatal Med 2007;12:273-279. 


\section{Titles and legends to figures}

\section{Fig 1. Immature hippocampal cells are sensitive to glucocorticoids.}

(A) After 7 days in vitro (DIV), primary hippocampal cultures, derived from postnatal rats aged 4 days, were comprised of $\sim 40 \%$ neural precursor cells (NPC, labeled with anti-nestin) and $\sim 35 \%$ immature neurons (stained with anti-TuJ1 or -doublecortin [DCX]; $<10 \%$ of the cells stained with anti-NeuN, a marker of young neurons (NeuN), and $<20 \%$ of the cells were astrocytes and oligodendrocytes (stained with antibody O4). (B) Immunoreactive glucocorticoid receptor (GR) was localized in both NPC and neuronal progenitors; shown are the percentage of GR-expressing cells in the different cell populations. NPC (nestin-positive cells identified by immunocytochemistry or cells transfected with nestin-GFP) and immature neurons (stained with anti-DCX or -TuJ1, or transfected with Ta-tubulin-GFP), as well as neurons (stained with anti-NeuN). The inset is an example of an immunoblot of cytoplasmic and nuclear fractions probed with GR antibody; note the increased GR signal in the nucleus (vs. cytoplasm) in lysates from cells that had been treated with glucocorticoid (dexamethasone, DEX, $10^{-5} \mathrm{M}$ ). Representative images of nestin-GFP-transfected and anti-nestin-stained cells co-expressing GR are shown; also shown are images from the same sets of cells after staining of the cell nuclei with Hoechst dye 33342; arrowheads point to identical cells in each row. (D) The dose-dependent induction of apoptosis by DEX is shown in the left-hand panel; these results are based on terminal deoxynucleotidyl transferase-mediated dUTP nick end-labeling (TUNEL) of apoptotic cells. Consistently robust responses are obtained at a dose of $10^{-5} \mathrm{M}$ (used in all subsequent experiments). The right-hand panel shows that similar results are obtained when apoptosis is evaluated by either TUNEL histochemistry or immunocytochemistry for the active (cleaved) form of the executioner caspase, capsase 3 . Note that the 
apoptotic actions of DEX can be significantly attenuated by pretreatment (30 min) of cells with the GR antagonist RU38486 $\left(10^{-5} \mathrm{M}\right)$, indicating mediation by GR. All numerical data are depicted as mean \pm SD. ${ }^{*} \mathrm{P}<0.05$ vs. CON, \# $\mathrm{P}<0.05$ vs. DEX. Scale bar $=50 \mu \mathrm{m}$ in $(\mathbf{A}), 20 \mu \mathrm{m}$ in (C).

Fig 2. Proliferating and resting cells are targets of GC-induced apoptosis.

(A) Exposure of DIV7 hippocampal cultures to DEX $\left(10^{-5} \mathrm{M}\right)$ leads to a significant loss of NPC (nestin-positive) and immature neurons (DCX/TuJ1-positive). (B) and (C), Representative images of cells that were treated simultaneously with $\operatorname{BrdU}(20 \mu \mathrm{M})$ and $\operatorname{DEX}\left(10^{-5} \mathrm{M}\right)$ before staining $24 \mathrm{~h}$ later for BrdU (to mark cells born in the preceding $24 \mathrm{~h}$ ) and TUNEL. TUNEL-labeling is shown in control (B1) and DEX-treated cells (C1), respectively; panels B2-B5 and C2-C5 are higher magnifications of the areas marked by the dotted boxes in B1 and C1, respectively; B2 and C2 show apoptotic cells, B3 and C3 show BrdU-incorporating cells, and B4 and C4 show nuclear staining with Hoechst dye, in control and DEX-treated cells, respectively; panels B5 and C5 show merged images of B2-B4 and C2-C4, respectively. Arrowheads point to identical cells in each column and exemplify apoptosis (TUNEL-stained) in recently-proliferated cells (BrdU-stained). (D) Quantitative analysis of TUNEL staining in proliferative (BrdU+) and resting (BrdU-) cells. (E) Comparable data to those shown in (D) were obtained when cells were double-labeled for $\mathrm{BrdU}$ and cleaved (active) caspase 3. All numerical data are shown as mean $\pm S D$. Asterisks indicate significant differences vs. control (untreated) cells $(P<$ 0.05). Scale bars: $20 \mu \mathrm{m}$.

Fig 3. Neural precursors and neuronal progenitors are driven into apoptosis by DEX.

Cultures were transfected with either nestin-GFP or Ta-tubulin-GFP plasmids to label neural precursors 


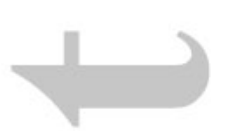

or neuronal progenitors, respectively. The percentage of cells staining positively for TUNEL or activated caspase 3 among the nestin- or Ta-tubulin-labeled populations were counted to examine how each phenotype was influenced by DEX. Representative images from TUNEL-stained cells that had been previously transfected with nestin-GFP (A-C and G-I) or Ta-tubulin-GFP (E-F and J-L); control cells are shown in A-C and D-F and DEX-treated cells are shown in G-I and $\mathbf{J}-\mathbf{L}$, where solid arrowheads indicate apoptotic GFP+ cells, detected by TUNEL and Hoechst staining. Examples of activated caspase 3 staining in specifically tagged NPC (nestin-GFP) and neuronal progenitor (Ta-tubulin-GFP) sub-populations are shown in $\mathbf{M}-\mathbf{T}$; open arrowheads indicate activated caspase3-/GFP+ cells and solid arrowheads point to activated caspase3+/GFP+ cells. Numerical analysis of these data is shown in $\mathbf{U}$ and V. Note that astrocytes labeled with GFAP-GFP do not undergo DEX-induced apoptosis (U). All numerical data are given as mean \pm SD. ${ }^{*} P<0.05$ vs. CON, \# $P<0.05 v s$. DEX. Scale bars: $20 \mu \mathrm{m}$.

Fig 4. DEX induces NPC apoptosis by increasing ROS production, perturbation of the mitochondrial membrane potential and subsequently, activation of the intrinsic apoptotic pathway.

(A) Pretreatment with either Ac-DEVD-cmk (caspase 3 inhibitor) or Ac-LEHD-cmk (caspase 9 inhibitor) rescue NPC from DEX-induced apoptosis. (B) Expression levels of Bcl-2 family members were measured by qPCR after exposure of cultures to various doses of DEX; dose response curves, in terms of the ratio of pro-apoptotic bax to anti-apoptotic $b c l-2$ or $b c l-x l$, reveal that DEX first produces a significant increase in the bax:bcl-2 ratio at a dose of $10^{-5} \mathrm{M}$, and that ratio of bax:bcl-xl is not influenced by DEX treatment. (C) Treatment of NPC cultures with DEX $\left(10^{-5} \mathrm{M}\right)$ stimulates ROS production, indicated by the intercalation of ethidum into DNA (red fluorescence). (D) Confirmation of 
DEX-stimulated ROS production in identified NPC that were labeled with nestin-GFP. (E) Treatment of primary cultures with DEX increases ROS production, as measured by DHE staining; note NPC, marked with nestin-GFP also show significantly increased levels of ROS in response to DEX. (F) Immunostaining for the p47-phox, a subunit of nicotinamide adenine dinucleotide phosphate (NADPH) oxidase, showing localization of the signal from the cytoplasm to the plasma membrane (arrowheads) in NPC (identified by nestin immunostaining) after DEX treatment. (G) Treatment of cultures with DEX leads to significant reductions in two key anti-oxidant enzymes, glutathione (GSH) and superoxide dismutases (SOD); enzyme activities were normalized to protein concentrations of the cell extracts. All numerical data are depicted as mean $\pm \mathrm{SD} .{ }^{*} \mathrm{P}<0.05 \mathrm{vs}$. CON, \# $\mathrm{P}<0.05 \mathrm{vs}$. DEX. Scale bar $=20 \mu \mathrm{m}$ in $\mathbf{C}, 10 \mu \mathrm{m}$ in $\mathbf{D}$ and $\mathbf{F}$.

Fig 5. Neonatal GC treatment induces apoptosis in the SGZ in situ.

(A) Immunohistochemistry for GR in the dentate gyrus of PND10 rats revealed a gradient of staining intensity, rising from the germinative subgranular zone (SGZ) to the granule cell layer (GCL); the area enclosed by the white box is enlarged in the inset, where the arrowheads mark examples of GR-positive NPC at the hilus-SGZ border. (B) and (C), Confocal images of activated caspase 3 immunostaining in a section from the dorsal portion of the dentate gyrus of a control (B) and a DEX-treated (C) rat (PND10); the arrowheads point to examples of cells showing immunoreactivity in the SGZ. (D) Numerical analysis of sections from control $(n=7)$ and DEX-treated rats $(n=8)$ stained for activated caspase 3 activity in SGZ; data shown are mean \pm SEM. ${ }^{*} \mathrm{P}<0.05$ vs. CON. Scale bar: $1 \mathrm{~mm}$ in $(\mathbf{A})$ and $20 \mu \mathrm{m}$ in the inset of $(\mathbf{A})$, (B) and (C). 
Fig 6. GC treatment in neonatal life results in a sustained reduction of neurogenic capacity.

(A)-(C), Confocal images showing double staining with anti-BrdU (A) and anti-Ki67 (B). Arrowheads indicate cells co-labeled with the BrdU and Ki67 antibodies. Hoechst 33342 staining (C) was used to identify cell nuclei and to help delineate the SGZ and GCL. (D) DEX treatment (200 $\mu \mathrm{g} / \mathrm{kg} / \mathrm{d}$ on PND1-3, tapering to $100 \mu \mathrm{g} / \mathrm{kg} / \mathrm{d}$ ) on PND1-7 results in a significant reduction in the number of proliferating cells, as judged by immunostaining of BrdU-incorporating cells and Ki67-immunoreactive cells on PND10, 18 and 28; note the progressive decline in proliferating cells in controls over the time period examined. (E) The volume of the SGZ and GCL of rats exposed to DEX on PND1-7 is significantly smaller when estimated on PND10 and 28. Note that the increase in GCL volume, between PND10 and PND28, was greater in controls than in neonatally DEX-treated rats. All numerical values are mean $\pm S E M$. ${ }^{*} P<0.05$, ${ }^{* *} \mathrm{P}<0.01$, as compared to age-matched controls; \#\# indicates significant difference between P10 and P28 (P<0.01). PND10, CON, $n=7$; DEX, $n=8 ;$ PND 18, CON, $n=8, ; D E X, n=8 ;$ PND28, CON, $n=8 ;$ DEX, $\mathrm{n}=9$. Scale bar: $20 \mu \mathrm{m}$.

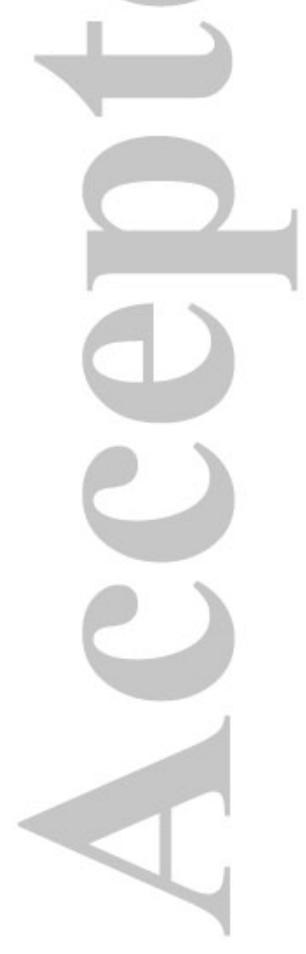



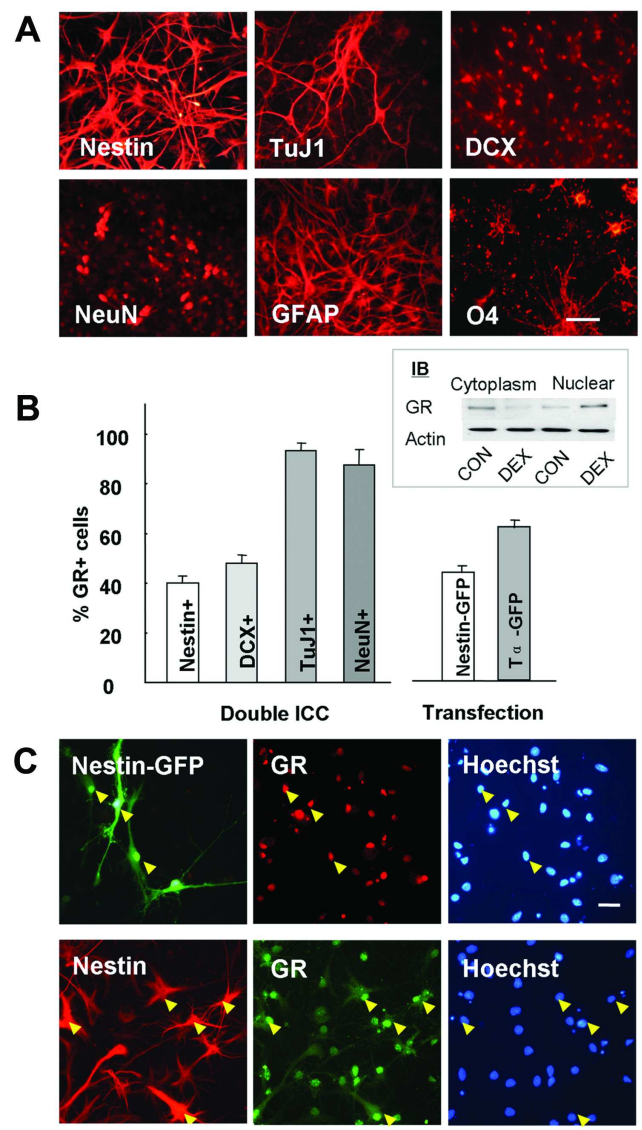

D
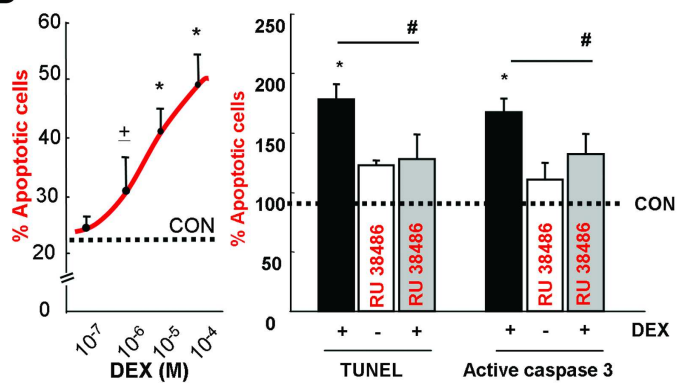

Fig 1. Immature hippocampal cells are sensitive to glucocorticoids.

(A) After 7 days in vitro (DIV), primary hippocampal cultures, derived from postnatal rats aged 4 days, were comprised of 40\% neural precursor cells (NPC, labeled with anti-nestin) and 35\% immature neurons (stained with anti-Tu]1 or -doublecortin [DCX]; $<10 \%$ of the cells stained with

anti-NeuN, a marker of young neurons (NeuN), and $<20 \%$ of the cells were astrocytes and oligodendrocytes (stained with antibody O4). (B) Immunoreactive glucocorticoid receptor (GR) was localized in both NPC and neuronal progenitors; shown are the percentage of GR in NPC (nestinpositive cells identified by immunocytochemistry or cells transfected with nestin-GFP) and immature neurons (stained with anti-DCX or-TuJ1, or transfected with Ta-tubulin-GFP), as well as neurons (stained with anti-NeuN). The inset is an example of an immunoblot of cytoplasmic and nuclear fractions probed with GR antibody; note the increased GR signal in the nucleus (vs. cytoplasm) in lysates from cells that had been treated with glucocorticoid (dexamethasone, DEX, 10-5 M). (C) Representative images of nestin-GFP-transfected and anti-nestin-stained cells co-expressing GR are 
shown; also shown are images from the same sets of cells after staining of the cell nuclei with Hoechst dye 33342; arrowheads point to identical cells in each row. (D) The dose-dependent induction of apoptosis by DEX is shown in the left-hand panel; these results are based on terminal deoxynucleotidyl transferase-mediated dUTP nick end-labeling (TUNEL) of apoptotic cells.

Consistently robust responses are obtained at a dose of 10-5 $\mathrm{M}$ (used in all subsequent experiments). The right-hand panel shows that similar results are obtained when apoptosis is evaluated by either TUNEL histochemistry or immunocytochemistry for the active (cleaved) form of the executioner caspase, capsase 3 . Note that the apoptotic actions of DEX can be significantly attenuated by pretreatment (30 min) of cells with the GR antagonist RU38486 (10-5 M), indicating mediation by GR. All numerical data are depicted as mean \pm SD. $* P<0.05$ vs. CON, \# $P<0.05$ vs. DEX. Scale bar $=50 \mu \mathrm{m}$ in (A), $20 \mu \mathrm{m}$ in (C).

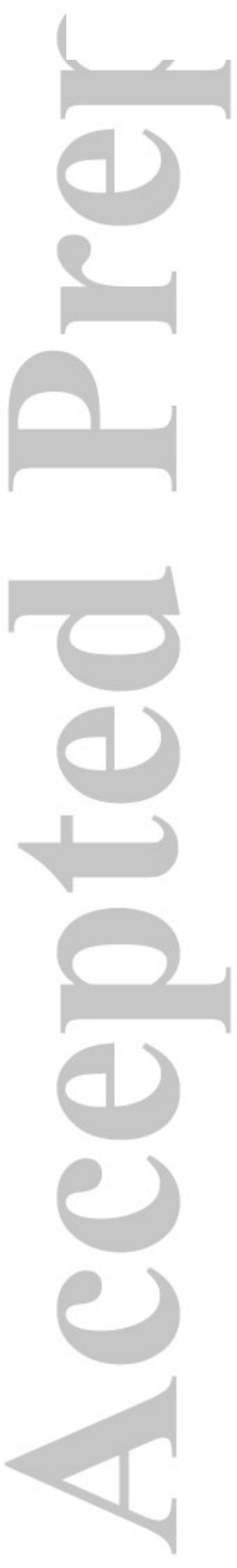

$70 \times 155 \mathrm{~mm}(600 \times 600 \mathrm{DPI})$ 

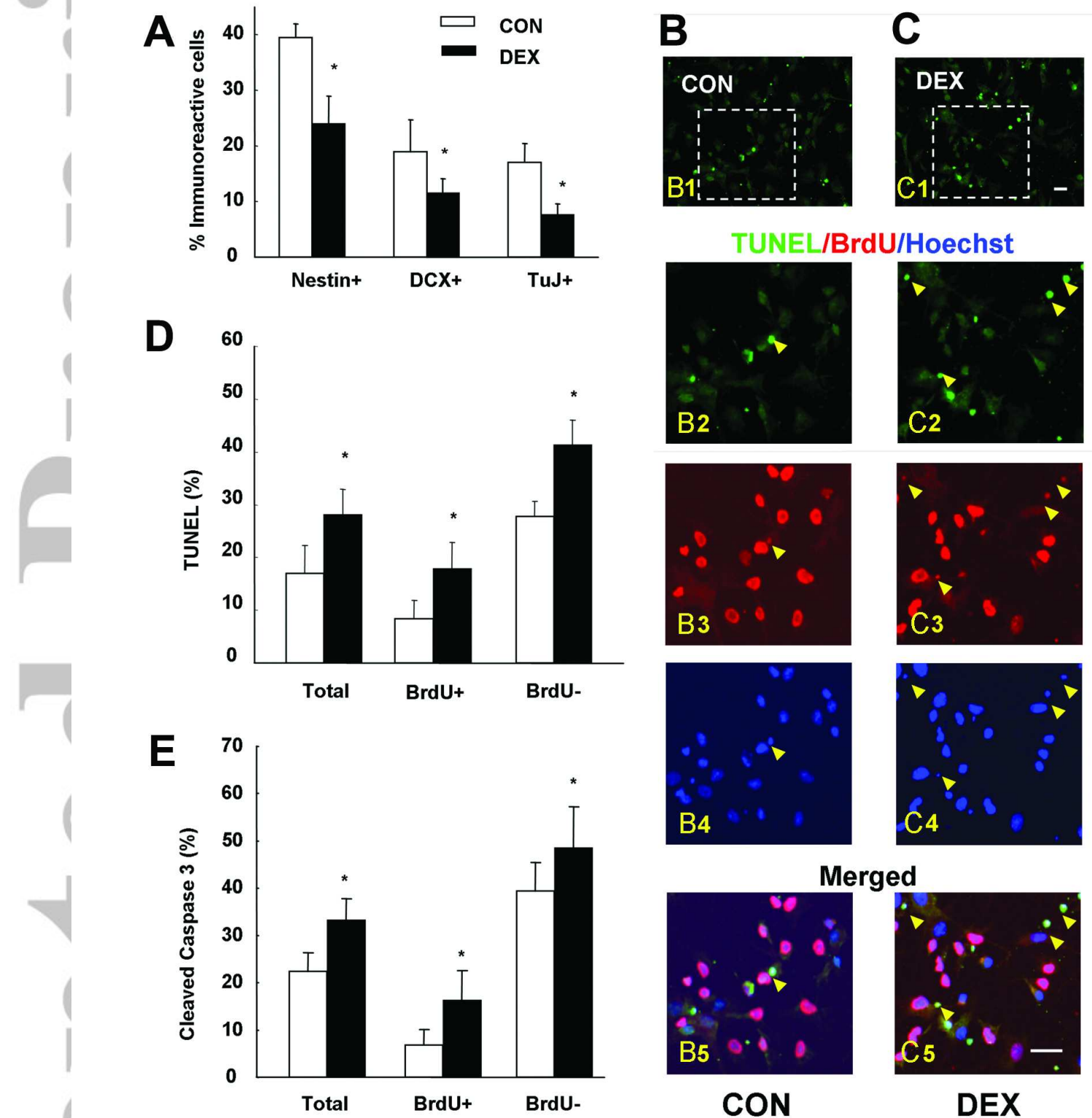

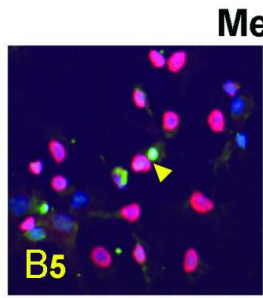

CON

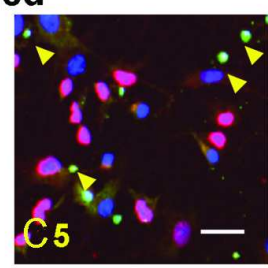

DEX

Fig 2. Proliferating and resting cells are targets of GC-induced apoptosis.

(A) Exposure of DIV7 hippocampal cultures to DEX (10-5 M) leads to a significant loss of NPC (nestin-positive) and immature neurons (DCX/TuJ1-positive). (B) and (C), Representative images of cells that were treated simultaneously with BrdU $(20 \mu \mathrm{M})$ and DEX $(10-5 \mathrm{M})$ before staining $24 \mathrm{~h}$ later for BrdU (to mark cells born in the preceding $24 \mathrm{~h}$ ) and TUNEL. TUNEL-labeling is shown in

control (B1) and DEX-treated cells (C1), respectively; panels B2-B5 and C2-C5 are higher magnifications of the areas marked by the dotted boxes in B1 and C1, respectively; B2 and C2 show apoptotic cells, B3 and C3 show BrdU-incorporating cells, and B4 and C4 show nuclear staining with Hoechst dye, in control and DEX-treated cells, respectively; panels B5 and C5 show merged images of B2-B4 and C2-C4, respectively. Arrowheads point to identical cells in each column and exemplify apoptosis (TUNEL-stained) in recently-proliferated cells (BrdU-stained). (D) Quantitative analysis of TUNEL staining in proliferative (BrdU+) and resting (BrdU-) cells. (E) Comparable data to those 

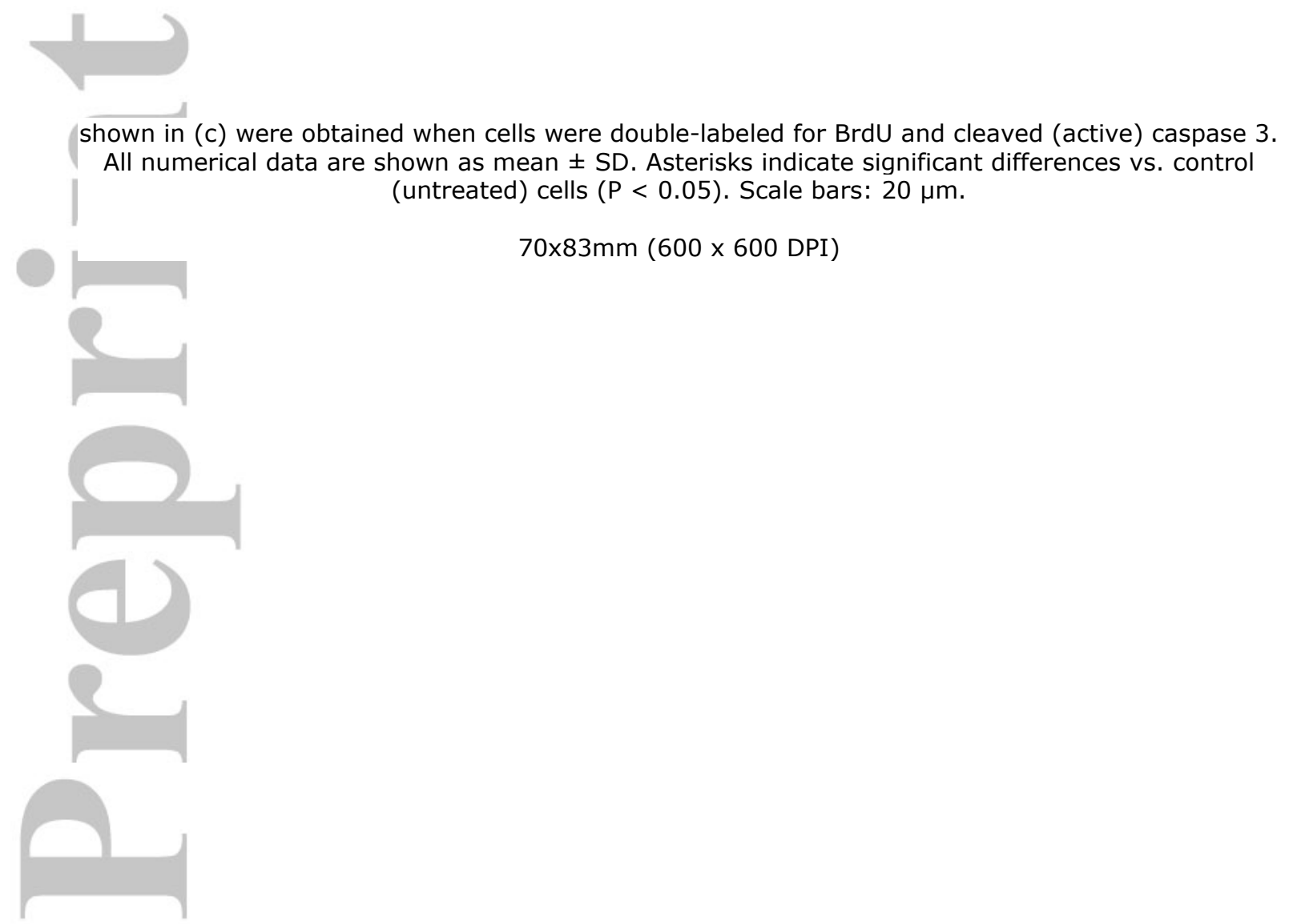

$70 \times 83 \mathrm{~mm}(600 \times 600 \mathrm{DPI})$

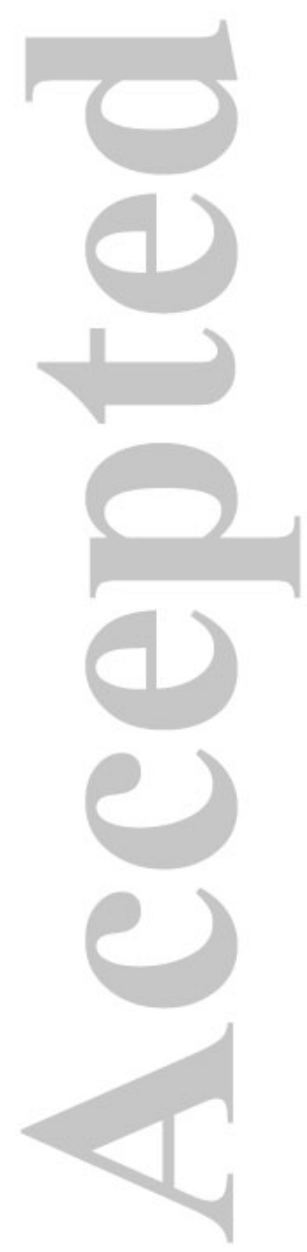



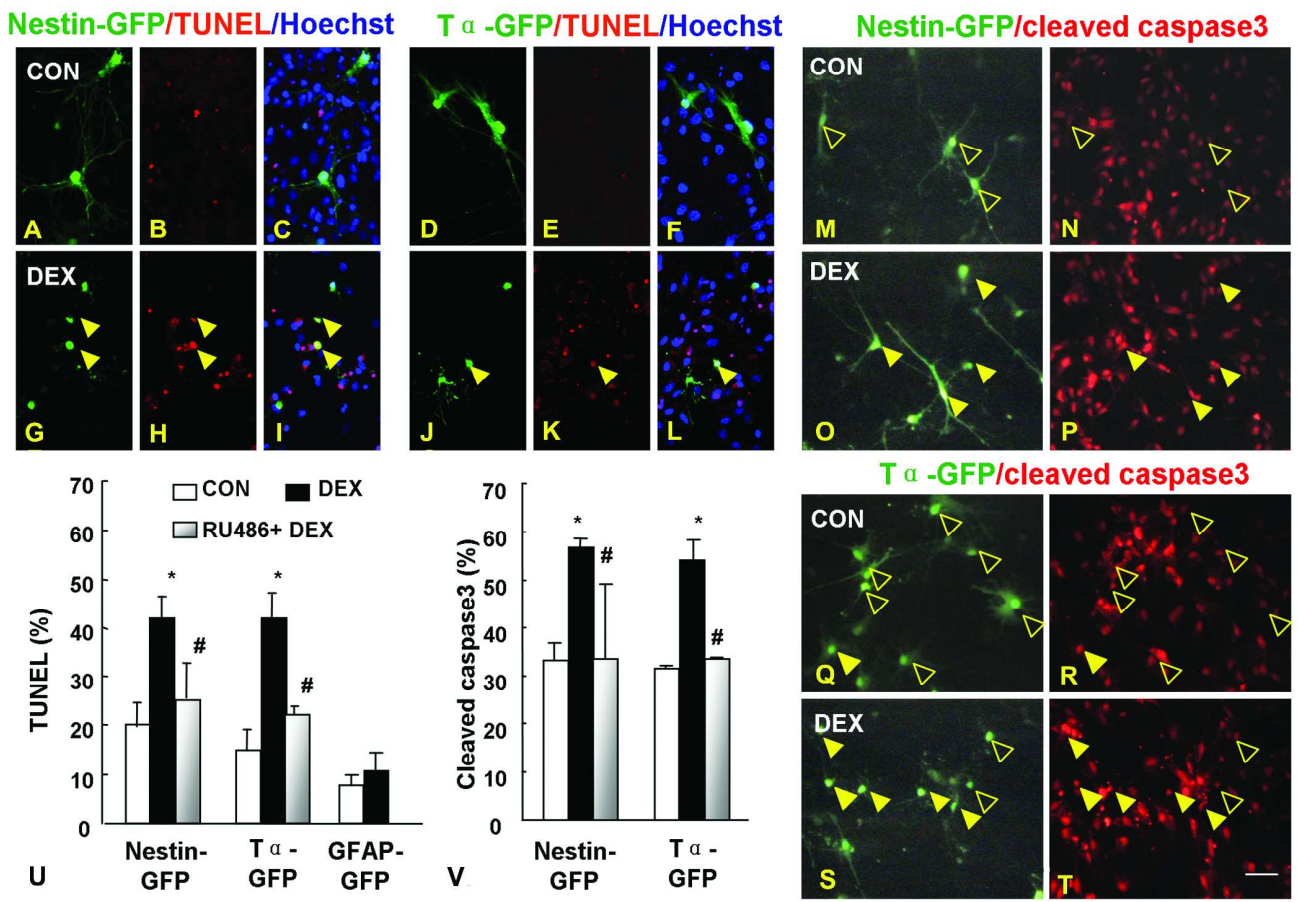

Fig 3. Neural precursors and neuronal progenitors are driven into apoptosis by DEX.

Cultures were transfected with either nestin-GFP or Ta-tubulin-GFP plasmids to label neural precursors or neuronal progenitors, respectively. The percentage of cells staining positively for TUNEL or activated caspase 3 among the nestin- or Ta-tubulin-labeled populations were counted to examine how each phenotype was influenced by DEX. Representative images from TUNEL-stained cells that had been previously transfected with nestin-GFP (A-C and G-I) or Ta-tubulin-GFP (E-F and $\mathrm{J}-\mathrm{L}$ ); control cells are shown in A-C and D-F and DEX-treated cells are shown in G-I and J-L, where solid arrowheads indicate apoptotic GFP+ cells, detected by TUNEL and Hoechst staining. Examples of activated caspase 3 staining in specifically tagged NPC (nestin-GFP) and neuronal progenitor (Tatubulin-GFP) sub-populations are shown in M-T; open arrowheads indicate activated caspase3/GFP+ cells and solid arrowheads point to activated caspase3+/GFP+ cells. Numerical analysis of these data is shown in $U$ and $V$. Note that astrocytes labeled with GFAP-GFP do not undergo DEXinduced apoptosis (U). All numerical data are given as mean \pm SD. $* P<0.05$ vs. CON, \# $P<$ 0.05 vs. DEX. Scale bars: $20 \mu \mathrm{m}$.

$178 \times 125 \mathrm{~mm}(600 \times 600 \mathrm{DPI})$ 
A

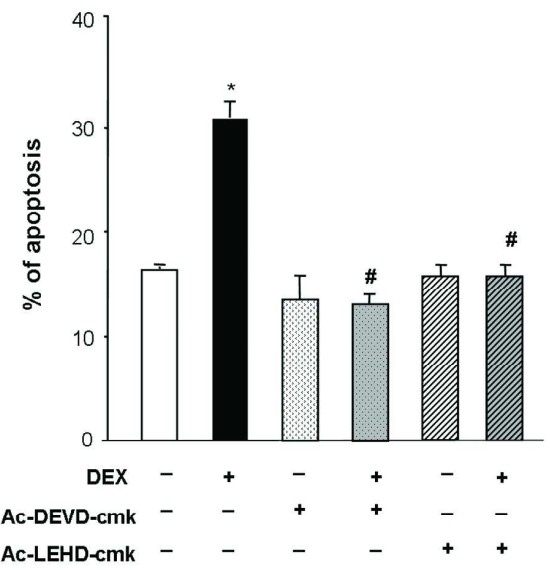

B

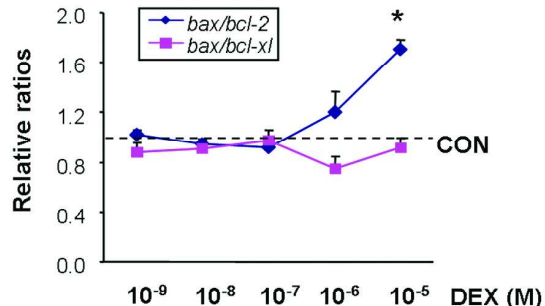

F
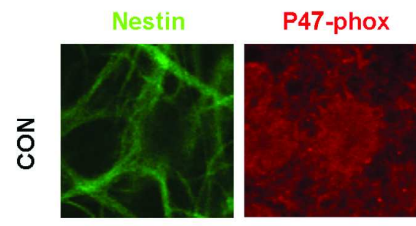

Merge

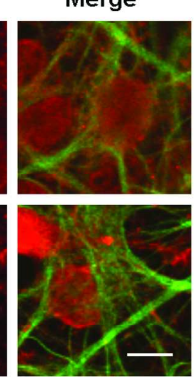

C
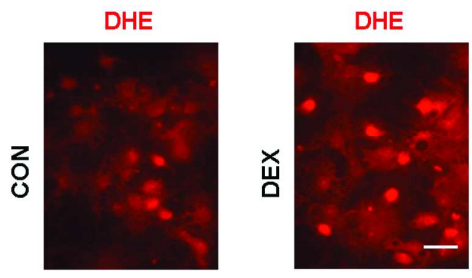

D

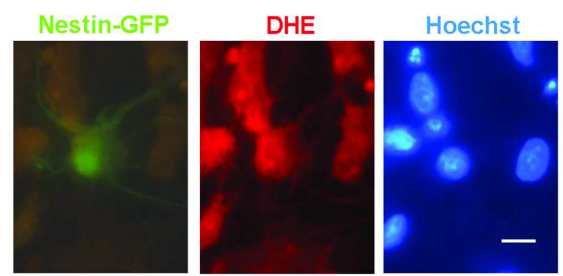

$\mathrm{E}$

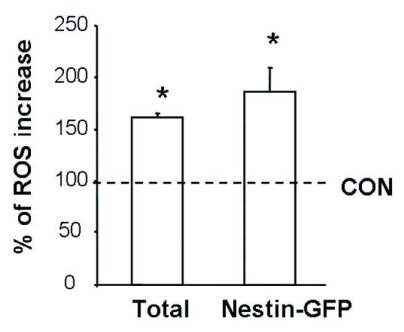

G

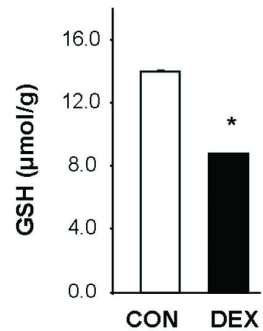

Fig 4. DEX induces NPC apoptosis by increasing ROS production, perturbation of the mitochondrial membrane potential and subsequently, activation of the intrinsic apoptotic pathway.

(A) Pretreatment with either Ac-DEVD-cmk (caspase 3 inhibitor) or Ac-LEHD-cmk (caspase 9 inhibitor) rescue NPC from DEX-induced apoptosis. (B) Expression levels of Bcl-2 family members were measured by qPCR after exposure of cultures to various doses of DEX; dose response curves,

in terms of the ratio of pro-apoptotic bax to anti-apoptotic bcl-2 or bcl-xl, reveal that DEX first produces a significant increase in the bax:bcl-2 ratio at a dose of 10-5 $\mathrm{M}$, and that ratio of bax: bcl$\mathrm{XI}$ is not influenced by DEX treatment. (C) Treatment of NPC cultures with DEX (10-5 M) stimulates

ROS production, indicated by the intercalation of ethidum into DNA (red fluorescence). (D)

Confirmation of DEX-stimulated ROS production in identified NPC that were labeled with nestin-GFP.

(E) Treatment of primary cultures with DEX increases ROS production, as measured by DHE

staining; note NPC, marked with nestin-GFP also show significantly increased levels of ROS in response to DEX. (F) Immunostaining for the p47-phox, a subunit of nicotinamide adenine

dinucleotide phosphate (NADPH) oxidase, showing localization of the signal from the cytoplasm to

the plasma membrane (arrowheads) in NPC (identified by nestin immunostaining) after DEX

treatment. (G) Treatment of cultures with DEX leads to significant reductions in two key anti-

oxidant enzymes, glutathione (GSH) and superoxide dismutases (SOD); enzyme activities were

normalized to protein concentrations of the cell extracts. All numerical data are depicted as mean \pm SD. $* \mathrm{P}<0.05$ vs. CON $\# \mathrm{P}<0.05$ vs. DEX. Scale bar $=20 \mu \mathrm{m}$ in $\mathrm{C}, 10 \mu \mathrm{m}$ in $\mathrm{D}$ and $\mathrm{F}$. 


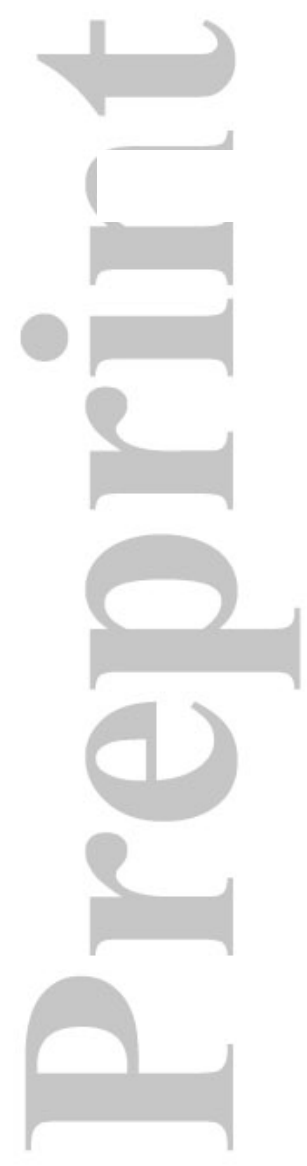

$180 \times 187 \mathrm{~mm}(600 \times 600 \mathrm{DPI})$

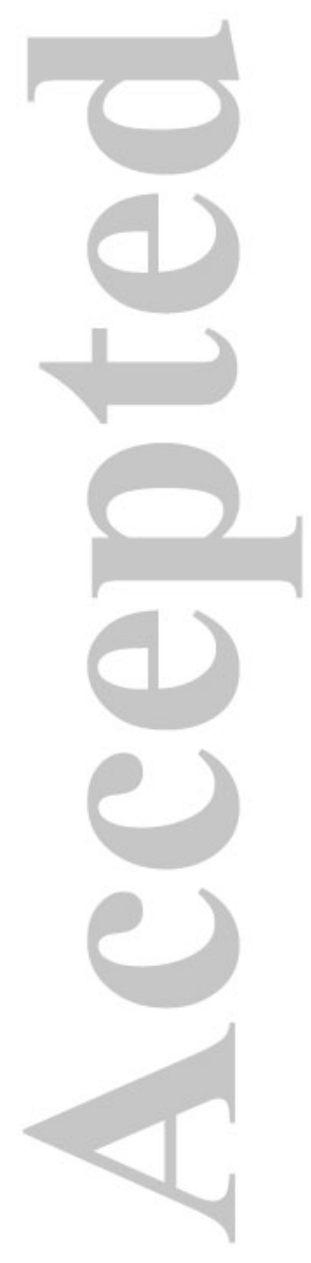

John Wiley \& Sons 


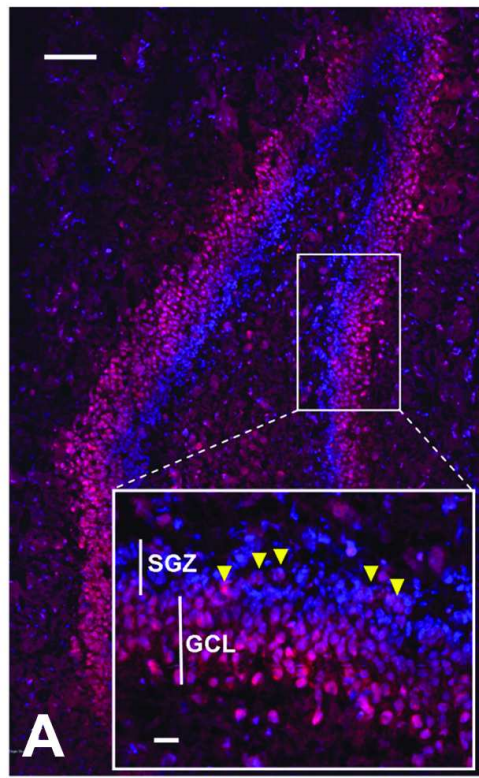

GR/Hoechst

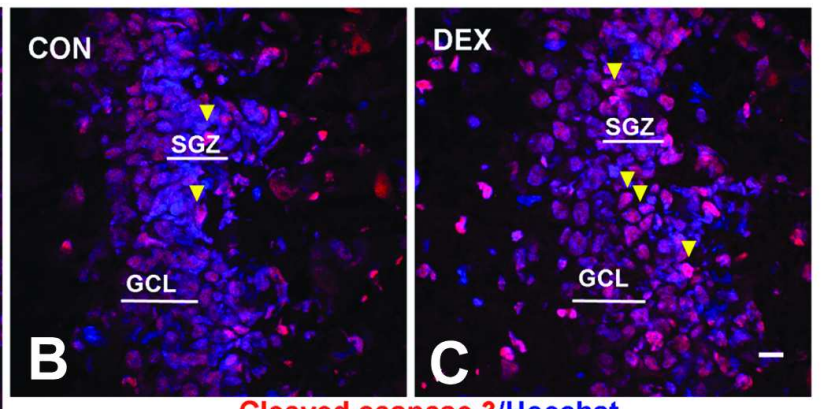

Cleaved caspase $3 /$ Hoechst

D

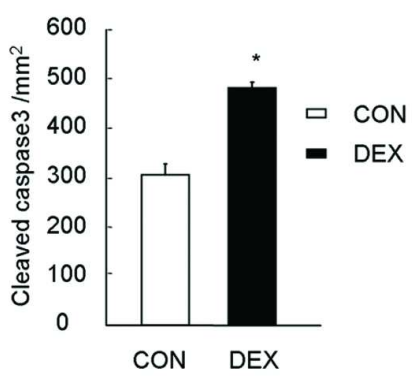

Fig 5. Neonatal GC treatment induces apoptosis in the SGZ in situ.

(A) Immunohistochemistry for GR in the dentate gyrus of PND10 rats revealed a gradient of staining intensity, rising from the germinative subgranular zone (SGZ) to the granule cell layer (GCL); the area enclosed by the white box is enlarged in the inset, where the arrowheads mark examples of GR-positive NPC at the hilus-SGZ border. (B) and (C), Confocal images of activated caspase 3 immunostaining in a section from the dorsal portion of the dentate gyrus of a control $(B)$ and a DEX-treated (C) rat (PND10); the arrowheads point to examples of cells showing immunoreactivity in the SGZ. (D) Numerical analysis of sections from control $(n=7)$ and DEX-treated rats $(n=8)$ stained for activated caspase 3 activity in SGZ; data shown are mean \pm SEM. $* P<0.05$ vs. CON. Scale bar: $1 \mathrm{~mm}$ in (A) and $20 \mu \mathrm{m}$ in the inset of $(A),(B)$ and $(C)$.

$$
70 \times 45 \mathrm{~mm}(600 \times 600 \mathrm{DPI})
$$



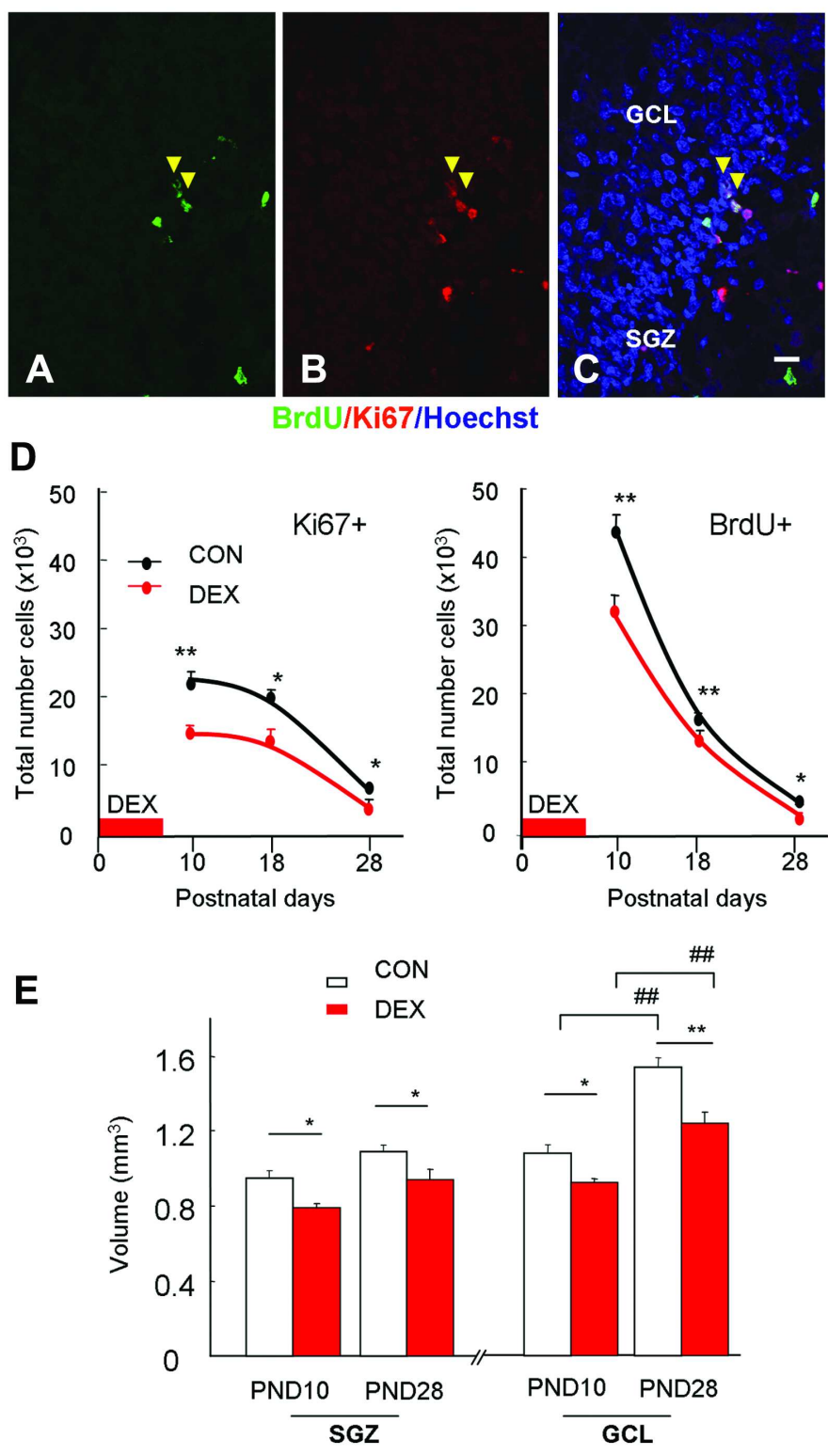

Fig 6. GC treatment in neonatal life results in a sustained reduction of neurogenic capacity. (A)-(C), Confocal images showing double staining with anti-BrdU (A) and anti-Ki67 (B). Arrowheads indicate cells co-labeled with the BrdU and Ki67 antibodies. Hoechst 33342 staining (C) was used to identify cell nuclei and to help delineate the SGZ and GCL. (D) DEX treatment $(200 \mu \mathrm{g} / \mathrm{kg} / \mathrm{d}$ on PND1-3, tapering to $100 \mu \mathrm{g} / \mathrm{kg} / \mathrm{d}$ ) on PND1-7 results in a significant reduction in the number of proliferating cells, as judged by immunostaining of BrdU-incorporating cells and Ki67-

immunoreactive cells on PND10, 18 and 28; note the progressive decline in proliferating cells in controls over the time period examined. (E) The volume of the SGZ and GCL of rats exposed to DEX on PND1-7 is significantly smaller when estimated on PND10 and 28. Note that the increase in GCL volume, between PND10 and PND28, was greater in controls than in neonatally DEX-treated rats. All numerical values are mean \pm SEM. $* \mathrm{P}<0.05$, ** $\mathrm{P}<0.01$, as compared to age-matched controls; \# \# indicates significant difference between P10 and P28 $(P<0.01)$. PND10, CON, $n=7 ; D E X, n=8$; 

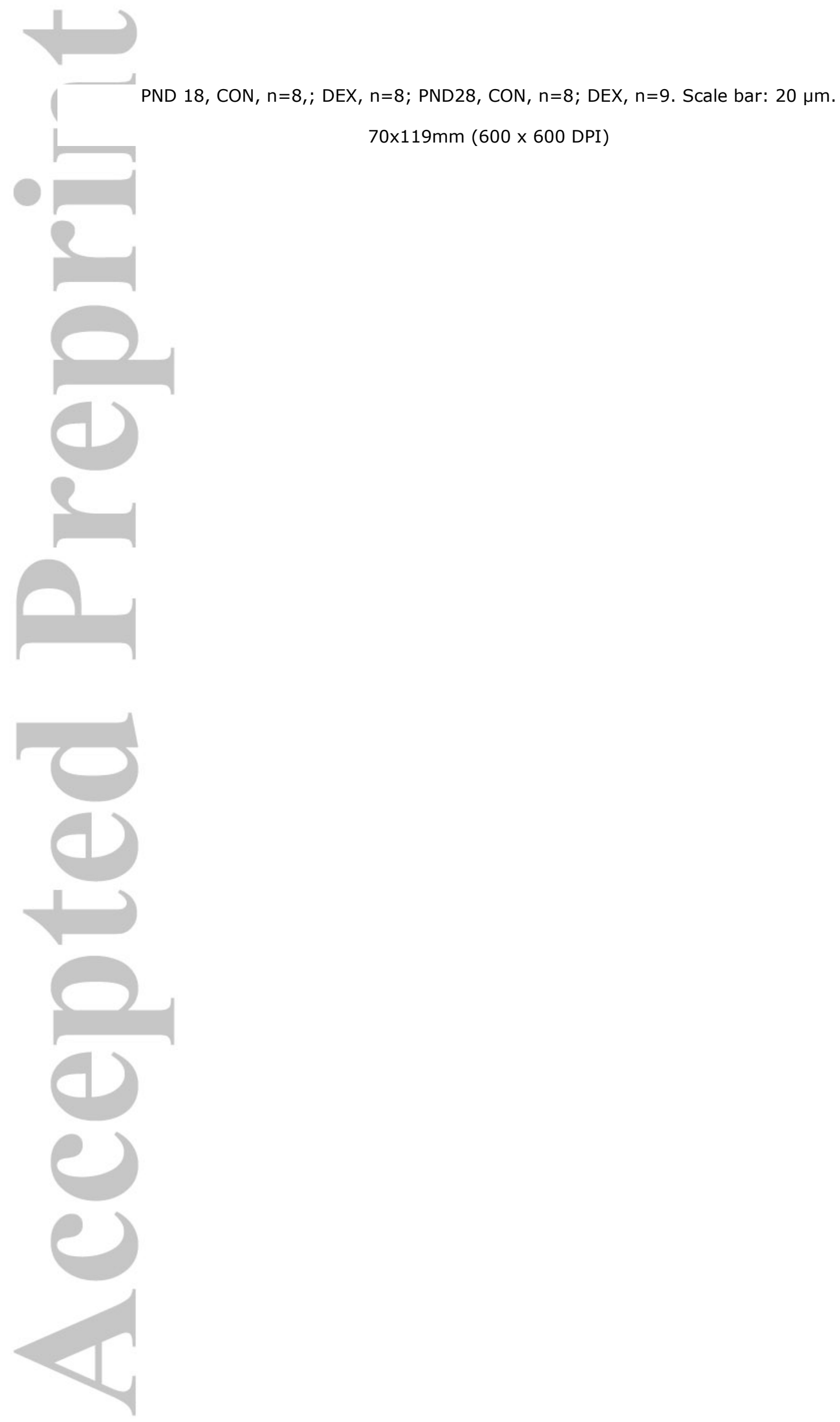


\section{SUPPLEMENTARY INFORMATION}

Yu et al. Depletion of the neural precursor cell pool by glucocorticoids

\section{Supplementary Methods}

Reactive oxygen species (ROS) generation: ROS generation was assayed by allowing dihydroethidium (DHE; $5 \mu \mathrm{M})$ to react $\left(30 \mathrm{~min} ; 37^{\circ} \mathrm{C}\right)$ with cellular superoxide ions, to yield red fluorescent ethidium. Cells were then washed and fixed in 4\% PFA before observation under a microscope (excitation, 520nm; emission, 590nm).

GSH and SOD assays: Glutathione (GSH) and superoxide dismutase (SOD) activity were measured using commercial GSH and SOD assay kits (Cayman Chemical, Tallinn, Estonia). Enzyme activities were corrected for protein concentration in the test samples.

Quantitative-PCR: Total RNA was isolated (RNAeasy kit; Qiagen, Hilden, Germany) and reverse transcribed with SUPERSCRIPTTM II RNA H-reverse transcriptase (Invitrogen) with customsynthesized Oligo-dT12-18 primers (MWG Biotech, Ebersberg, Germany). Quantitative PCR (qPCR) was performed using a LightCycler (Roche Diagnostics, Mannheim, Germany) in a volume of $10 \mu \mathrm{l}$ containing $2 \mu \mathrm{l}$ of $5 \mathrm{x}$ Faststart DNA SYBR green I (Roche) master mix, $5 \mu \mathrm{l}$ of water, $0.5 \mu \mathrm{l}$ of each primer, and $2 \mu \mathrm{l}$ of extracted DNA. Initial denaturation was carried out at $95^{\circ} \mathrm{C}$ (slope, $20^{\circ} \mathrm{C} / \mathrm{s}$ ) for 10 min, followed by 40 cycles of denaturation at $94^{\circ} \mathrm{C}(5 \mathrm{~s})$; annealing temperatures $(5 \mathrm{~s})$ were $63^{\circ} \mathrm{C}$ (for bcl-xL, bcl-2) and $64^{\circ} \mathrm{C}$ (for Bax and HPRT1), with an additional $10 \mathrm{~s}$ at $72^{\circ} \mathrm{C}$. The specific $5^{\prime}-3^{\prime}$ primer pair sequences were: Bax (174 bp), forward CTGCAGAGGATGATTGCTGA and reverse GATCAGCTCGGGCACTTTAG; bcl-xL (121bp), forward GATCAGCTCGGGCACTTTAG and reverse CAGGAACCAGCGGTTGAAA; bcl-2 (251bp), forward CGGTGGTGGAGGAACTCTTC and reverse CAGCCAGGAGAAATCAAACAGA; HPRT1 (203 bp), forward GGACCTCTCGAAGTGTTGGA and reverse GGCCACATCAACAGGACTCT. All calculations and statistical analyses were based on relative expression levels of the the mRNA of interest to levels of mRNA coding for the housekeeping gene HPRT1. 


\section{Supplementary legends}

Fig S1. Quiescent neural progenitor (QNP) and amplifying neural progenitor (ANP) cells express GR.

(A) Double immunostaining for nestin and GR in the subgranular zone (SGZ) of the dentate gyrus (PND6) revealed GR expression in nestin-positive NPCs; arrowheads indicate examples of cells showing both nestin and GR immunoreactivity. (B) Demonstration of colocalization of GR in GFAPand Sox2-positive cells within the dentate gyrus. The area enclosed by the white box is enlarged and shown in the right panel (B1 shows GFAP-, B2 shows GR-, B3 shows Sox2- immunostaining; B4 shows merged images). Hoechst staining was used to define the position of immunopositive cells. Open arrowheads indicate GR expression in ANP (GFAP-Sox2+), and solid arrowheads indicate GR expression in the QNP (GFAP+Sox2+). (C) Numerical representation of relative numbers of QNP and ANP cells that express GR; note the comparable expression of GR in both cell types. Data shown are mean \pm SD. Scale bar: $20 \mu \mathrm{m}$ in (A), (B1-B4) and $50 \mu \mathrm{m}$ in (B).

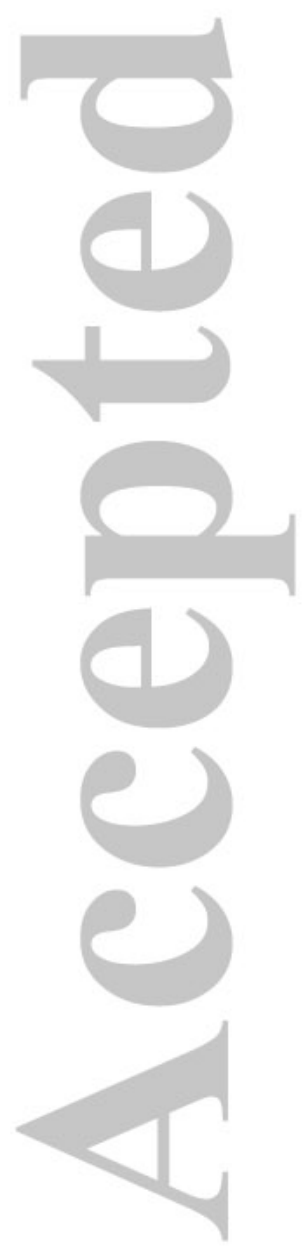




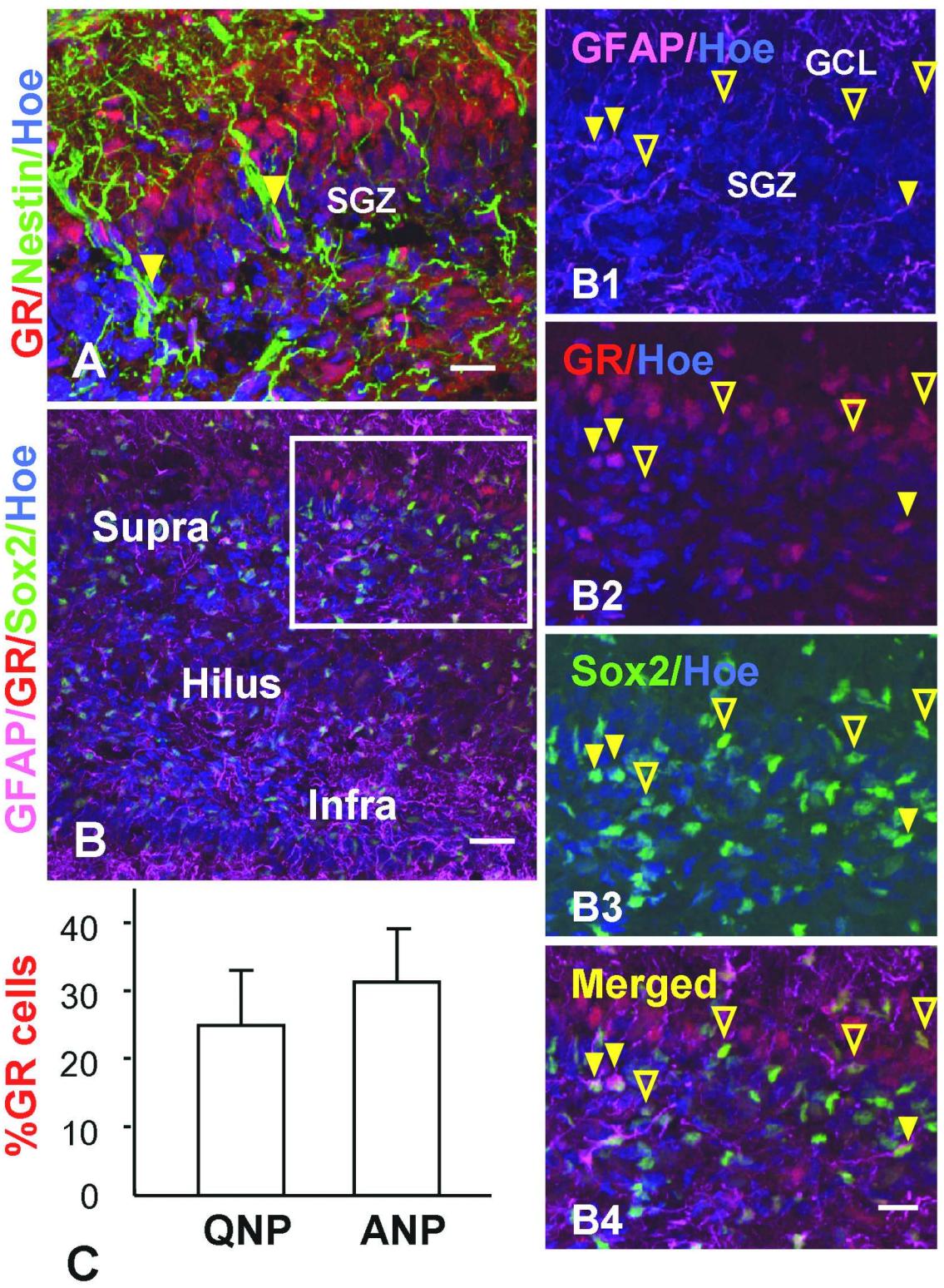

Fig S1. Quiescent neural progenitor (QNP) and amplifying neural progenitor (ANP) cells express GR. (A) Double immunostaining for nestin and GR in the subgranular zone (SGZ) of the dentate gyrus (PND6) revealed GR expression in nestin-positive NPCs; arrowheads indicate examples of cells showing both nestin and GR immunoreactivity. (B) Demonstration of colocalization of GR in GFAPand Sox2-positive cells within the dentate gyrus. The area enclosed by the white box is enlarged and shown in the right panel (B1 shows GFAP-, B2 shows GR-, B3 shows Sox2- immunostaining; B4 shows merged images). Hoechst staining was used to define the position of immunopositive cells. Open arrowheads indicate GR expression in ANP (GFAP-Sox2+), and solid arrowheads indicate GR expression in the QNP (GFAP+Sox2+). (C) Numerical representation of relative numbers of QNP and ANP cells that express GR; note the comparable expression of GR in both cell types. Data shown are mean \pm SD. Scale bar: $20 \mu \mathrm{m}$ in (A), (B1-B4) and $50 \mu \mathrm{m}$ in (B). 